\title{
Referees for Abstracts
}

Prof. Dr. M. Anlauf (Bremen)

Prof. Dr. C.A. Baldamus (Köln)

Prof. Dr. P. Baumgart (Münster)

Prof. Dr. M. Böhm (Köln)

Prof. Dr. G. Bönner (Bad Krozingen)

Prof. Dr. R. Bretzel (Giessen)

Prof. Dr. O.E. Brodde (Halle)

Prof. Dr. R. Dietz (Berlin)

Prof. Dr. A. Distler (Berlin)

Prof. Dr. R. Düsing (Bonn)

PD Dr. Th. Eschenhagen (Hamburg)

Prof. Dr. I.W. Franz (Todtmoos)

Prof. Dr. U. Frei (Berlin)

Prof. Dr. U. Gleichmann (Bad Oeynhaui

Prof. Dr. R. Griebenow (Köln)

Prof. Dr. H. Haller (Berlin)

Prof. Dr. K. Hayduk (Düsseldorf)

PD Dr. B. Heintz (Aachen)

Prof. Dr. H. Holzgreve (München)

Prof. Dr. J. Holtz (Halle)

Prof. Dr. H.U. Janka (Bremen)

Prof. Dr. E. Jungmann (Rheda-Wiedenbrück)

Prof. Dr. H. Kilbinger (Mainz)

Prof. Dr. R. Kolloch (Bielefeld)

Prof. Dr. B. Lemmer (Mannheim)

Prof. Dr. H. Liebau (Hamburg)

PD Dr. W. Linz (Frankfurt)

Prof. Dr. F.C. Luft (Berlin)

Prof. Dr. J. Mann (München)

Prof. Dr. M.C. Michel (Essen)

Prof. Dr. M. Middeke (Burg)

PD Dr. G.J. Molderings (Bonn)

Prof. Dr. W. Motz (Greifswald)

PD Dr. H. Pagel (Lübeck)

Prof. Dr. Th. Philipp (Essen)

Prof. Dr. M. Paul (Berlin)

Prof. Dr. K.H. Rahn (Münster)

Prof. Dr. R. Rettich (Greifswald)

PD Dr. G.H. Richardt (Lübeck) 
Prof. Dr. T. Risler (Tubingen) Prof. Dr. E. Ritz (Heidelberg) PD Dr. B. Schieffer (Hannover) Prof. Dr. R.E. Schmieder (Erlangen) Prof. Dr. J. Scholze (Berlin) Prof. Dr. J. Schrader (Cloppenburg) Prof. Dr. A.M. Sharma (Berlin) Prof. Dr. H.G. Siebert (Aachen) Prof. Dr. W. Siffert (Essen) Prof. Dr. G. Sponer (Mannheim) Prof. Dr. K.O. Stumpe (Bonn) PD Dr. P. Trenkwalder (Starnberg) Prof. Dr. Th. Unger (Kiel) Prof. Dr. H. Vetter (Bonn) Prof. Dr. W. Vetter (Zurich) Prof. Dr. G. Wiemer (Frankfurt) Prof. Dr. S. Willich (Berlin) Prof. Dr. W. Ziedek (Bochum) Prof. Dr. H. Zschiedrich (Bremen) 